\title{
A man with fever, cough, diarrhea and a coated tongue
}

$\mathrm{O}$ $\mathrm{n}$ a recent trip to Bolivia our team of visiting physicians was told of a rapidly spreading outbreak of an unidentified hemorrhagic fever, which local medical officials were treating as dengue. Over several days, about 600 people in the rural area developed different degrees of fever, headache, abdominal pain, occasional dry cough and epistaxis. At one point, nearly $25 \%$ of the approximately 3500 people living in the region were affected. After being invited by the local authorities to help investigate the illness, we began to interview and examine affected individuals.

One 60-year-old man's case was typical of many in the outbreak. This previously healthy farmer presented to his local medical clinic on day 10 with a week-long history of fever, abdominal pain, and mild, nonhemorrhagic diarrhea. In the previous 48 hours, a bothersome, nonproductive cough had also developed. On examination, the man was found to have vague epigastric tenderness but no organomegaly, mild rales heard at the base of both lungs and a "furry" tongue (Fig. 1). He had no appreciable rash, and the rest of his examination was unremarkable.

We suspected an outbreak of typhoid fever. Laboratory investigations were limited, but a Widal test (for agglutinating antibodies to $\mathrm{O}$ and $\mathrm{H}$ antigens of the Salmonella typhus bacterium) was performed in 10 of the most severe cases (including 5 children and the patient described here); the results were positive in 8 . The patients were given a fluoroquinolone. Because the source of the outbreak was yet unclear, much of our efforts were directed toward working with regional health officials to provide education to the population about handwashing, water iodination and the use of boiled water in food preparation. The mortality was low, but exact figures are unknown.

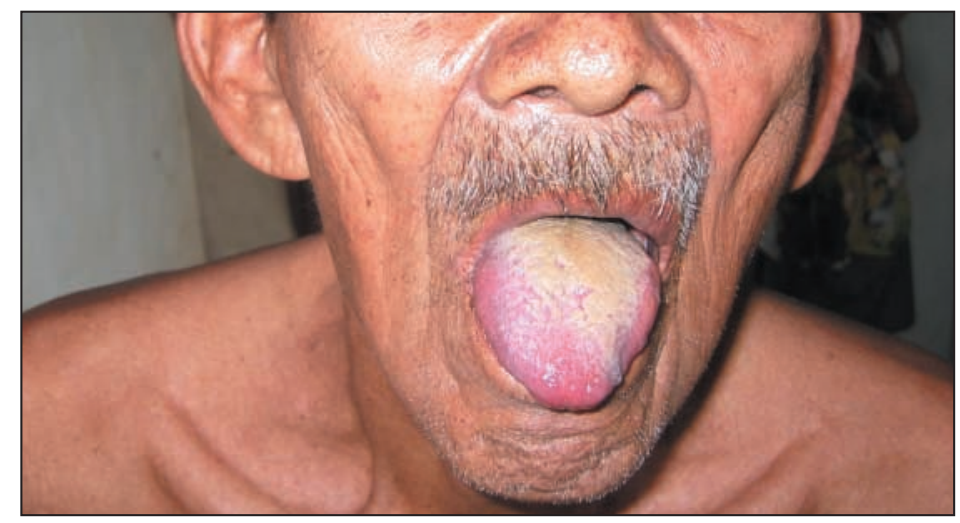

Typhoid fever is a systemic bacterial disease caused by S. typhi, an organism for which humans are the only host. ${ }^{1}$ It has been estimated that 16-17 million cases of typhoid fever occur annually worldwide, resulting in about 600000 deaths each year. ${ }^{1,2}$

Typically, there is a 7- to 14day asymptomatic period after ingestion of the bacterium, followed by onset of bacteremia marked by fever and malaise. ${ }^{1}$ Patients often present at the end of the first week with fever, dull headache, myalgias, fatigue and gastrointestinal symptoms, including anorexia, nausea and poorly localized abdominal pain. Constipation is almost as likely as diarrhea to be a symptom of this enteric illness. ${ }^{1,3,4}$ A nonproductive cough is common, and epistaxis may occur but is rare. Other signs and symptoms of typhoid fever include prolonged fever, relative bradycardia and a rash consisting of rose spots (pinkish lesions $1-4 \mathrm{~mm}$ in diameter) usually on the abdomen and chest. ${ }^{1,4} \mathrm{~A}$ fever exhibiting a stepladder rise over the first week, with a plateau in the second week and a gradual fall in the third or fourth week is typical. ${ }^{4}$ On examination, patients may have a slow, dicrotic pulse (tracing shows 2 peaks instead of the usual one, the second one coming during diastole as an exaggeration of the dicrotic wave).

Patients may become confused, and some may exhibit a toxic, or apathetic facies, which has been described as character- istic of typhoid. ${ }^{1,4}$ In addition to this facial sign, patients can have a furry, or coated, tongue, ${ }^{1,4,5}$ as seen in Fig. 1. In one study, ${ }^{5}$ coated tongue was found to be 1 of 4 specific clinical markers for typhoid fever (in addition to high fever, loose bowel movements and bradycardia), with a reported specificity of $94 \%$. Thus, the evaluation of any patient presenting with symptoms suggestive of typhoid fever warrants a thorough oropharyngeal examination, since a coated tongue, if present, may provide an important diagnostic clue in an otherwise nonspecific presentation.

\section{Sharon K. Bal}

Resident, Family Medicine

Charles Czarnowski

Assistant Professor

Department of Family Medicine

University of Ottawa

Ottawa, Ont.

\section{References}

1. Parry CM, Hien TT, Dougan G, White NJ, Farrar JJ. Typhoid fever. N Engl F Med 2002;347(22):1770-82.

2. World Health Organization. Typhoid fever [fact sheet no N149]. Geneva: The Organization; March 1997. Available: www.who.int/inf-fs/en/fact149 html (accessed 2004 Feb 24).

3. Yaramis Y, Yildirim I, Selahattin K, Nuri-Ozbek M, Yalcin I, Tas A et al. Clinical and laboratory presentation of typhoid fever. Int Pediatr 2001;16 (4):227-30.

4. Huckstep RL. Typhoid fever and other Salmonella infections. Edinburgh: E\&S Livingstone Ltd.; 1962. ch. 6-8. Available: www.worldortho.com/huckstep /typhoid (accessed 2004 Feb 24).

5. Haq SA, Alam MN, Hossain SM, Ahmed T, Tahir M. Value of clinical features in the diagnosis of enteric fever. Bangladesh Med Res Counc Bull 1997;23(2):42-6. 